\title{
Optimal Illumination of Phase-only Diffractive Element Using GPC Light shaper.
}

Villangca, Mark Jayson; Bañas, Andrew Rafael; Kopylov, Oleksii; Palima, Darwin; Glückstad, Jesper

Published in:

Proceedings of SPIE

Link to article, DOI:

$10.1117 / 12.2079118$

Publication date:

2015

Document Version

Publisher's PDF, also known as Version of record

Link back to DTU Orbit

Citation (APA):

Villangca, M. J., Bañas, A. R., Kopylov, O., Palima, D., \& Glückstad, J. (2015). Optimal Illumination of Phaseonly Diffractive Element Using GPC Light shaper. In Proceedings of SPIE (Vol. 9379). [937900] SPIE International Society for Optical Engineering. https://doi.org/10.1117/12.2079118

\section{General rights}

Copyright and moral rights for the publications made accessible in the public portal are retained by the authors and/or other copyright owners and it is a condition of accessing publications that users recognise and abide by the legal requirements associated with these rights.

- Users may download and print one copy of any publication from the public portal for the purpose of private study or research.

- You may not further distribute the material or use it for any profit-making activity or commercial gain

- You may freely distribute the URL identifying the publication in the public portal 


\title{
Optimal Illumination of Phase-only Diffractive Element Using GPC Light shaper
}

\author{
Mark Villangca*, Andrew Bañas, Oleksii Kopylov, Darwin Palima and Jesper Glückstad \\ DTU Fotonik, Dept. of Photonics Engineering, Technical University of Denmark \\ DK-2800 Kgs. Lyngby, Denmark \\ *Email: majv@fotonik.dtu.dk \\ www.ppo.dk
}

\begin{abstract}
We have previously proposed and demonstrated optimal beam shaping of contiguous light patterns using the Generalized Phase Contrast (GPC) method. The concept has been packaged into a compact add-on module, which we call the GPC light shaper (LS) that can be conveniently integrated to existing optical setups requiring optimal illumination of devices such as spatial light modulators (SLMs). In this work, we integrated the GPC LS into a holography setup to generate more intense focal spots and extended patterns. The output of the holography setup with the GPC LS is compared with a similar setup but using only hard-truncated beams. Our results show that, we get a $\sim 3 \mathrm{x}$ gain in intensity of the generated patterns when using GPC LS.
\end{abstract}

Keywords: Generalized Phase Contrast, holography, spatial light modulators

\section{INTRODUCTION}

Many optical tweezers array ${ }^{1,2}$ and custom optical landscapes (e.g. vortex beams ${ }^{3}$, non-diffracting beams ${ }^{4}$, accelerating beam ${ }^{5}$, etc.) are generated using computer-generated (CG) holography. Nowadays, CG holography uses programmable phase-only diffractive element to modify the incident wavefront such that a desired intensity distribution is obtained at some output plane. Recently, there is a strong interest in the application of holography in neurophotonics where simultaneous multi-site two-photon photolysis is used to uncage neurotransmitters ${ }^{6}$. Increasing the number of light spots will decrease peak intensities for each spot and this is crucial for two-photon polymerization of parallel structures ${ }^{7}$. In some cases, amplification of the input beam is necessary ${ }^{7}$. Since a high laser power is not always available, efficient illumination of the diffractive element is therefore necessary to utilize most of the available photons.

Among other things, one consideration in using phase-only diffractive element is geometry. Most diffractive elements are pixelated and consequently have a square or rectangular overall shape. The typical holography setup involves hardtruncation of the incident beam to match the shape of the modulation element and thereby waste photons. It is therefore desirable to have an efficient management of photons get the most out of available laser sources. This is extremely useful when one is working already on the upper limit of available laser source.

In this work, we integrate the GPC $\mathrm{LS}^{8}$ in a $\mathrm{CG}$ holography setup to reshape the incident Gaussian beam from the laser source to match the shape of the SLM using the parameters calculated in literature ${ }^{9,10}$. We generate focal spots and extended patterns and compared the result against using only hard-truncated Gaussian beams.

\section{METHODS}

\subsection{Generalized Phase Contrast Light Shaper}

The GPC Light shaper is a compact add-on module that performs beam shaping using the GPC method ${ }^{11}$ as shown in figure 1 . It consists of a phase mask that matches the geometry of the target device (e.g. SLMs) and adds a phase shift in the incident Gaussian beam. The phase shifted field is then Fourier transformed into the plane of the phase contrast filter (PCF) which creates a synthetic reference wave from the low spatial frequencies. A second lens is used to take another

\footnotetext{
Complex Light and Optical Forces IX, edited by Enrique J. Galvez, Jesper Glückstad,

David L. Andrews, Proc. of SPIE Vol. 9379, 937900 - (c) 2015 SPIE

CCC code: $0277-786 \mathrm{X} / 15 / \$ 18 \cdot$ doi: $10.1117 / 12.2079118$
}

Proc. of SPIE Vol. 9379 937900-1 
Fourier transform and the resulting pattern is a contiguous intensity distribution corresponding to the shape of the phase mask (with inversion due the use of another Fourier transform instead of the inverse).

The phase mask and the PCF are made from fused silica which is chemically etched to obtain the necessary phase shift. In the experiment we use a $\pi$ phase shift. The components of the GPC LS are assembled using mounts for half-inch optics from Thorlabs.

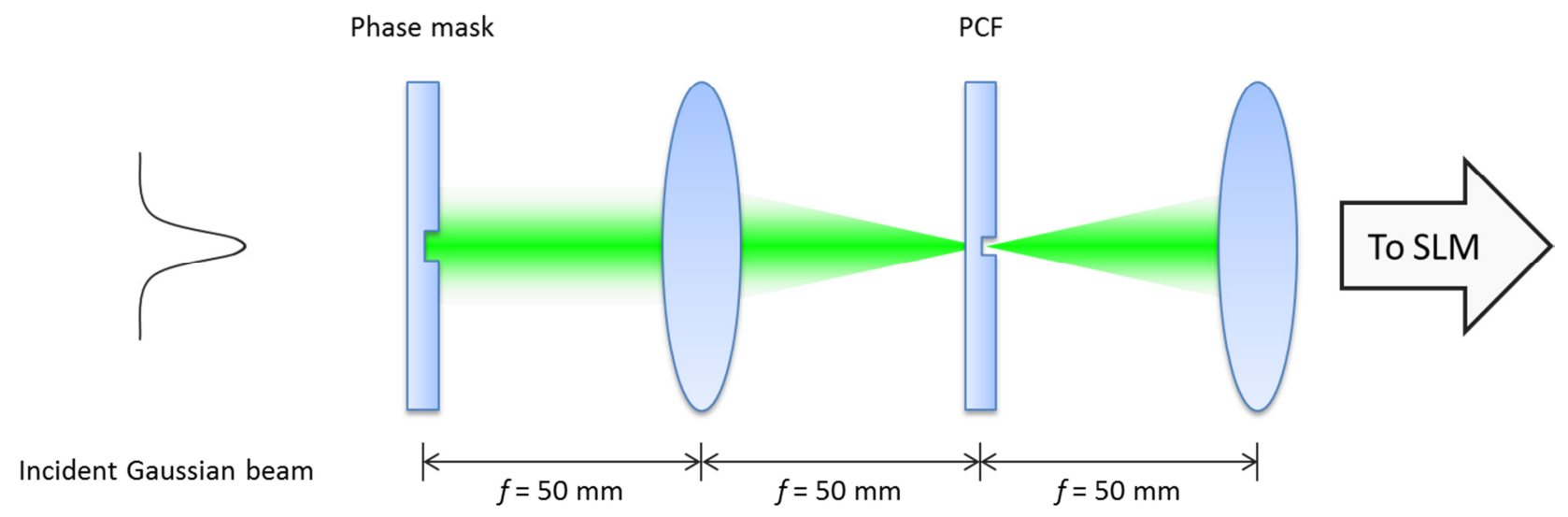

Figure 1. The GPC light shaper. An incident Gaussian beam is shaped according to the chosen phase mask. In the experiment, the phase mask is chosen to match the geometry of the SLM.

\subsection{Phase-only diffractive setup}

The experimental setup is shown in figure 2. It consists of a laser source (Laser Quantum Excel, $\lambda=532 \mathrm{~nm}$ ) with beam waist $2 w_{0}=1.5 \mathrm{~mm}$. The beam is de-magnified 1.5 times since the phase mask and PCF are designed for a $1 \mathrm{~mm}$ beam diameter. The phase mask has a 4:3 rectangular phase shifting region that matches the aspect ratio of the SLM. An iris is used to block some of the stray light and also serves as the aperture to create the hard-truncated beam. Further magnification is added to match the size of the SLM (Hamamatsu Photonics, $798 \times 600$ pixels, $9.9 \mathrm{~mm} \times 7.5 \mathrm{~mm}$ active area).

In the hard-truncated case, the phase mask and PCF is moved away from the path of the beam to ensure that the beam will encounter similar materials and magnification along the beam path. A beam profiler (Gentec-EO Beamage 3.0) is used to capture the resulting intensity distribution after taking the Fourier transform of the field modulated by the SLM.

The GPC-enhanced beam and hard-truncated beam are tested for random arrangement of focal spots and extended patterns. The holograms for the focal spots and extended patterns are calculated using the Gerchberg-Saxton algorithm ${ }^{12}$. Note that we did not perform suppression of the zero-order and higher-order diffraction. However, to maintain uniformity in the intensity of focal spots, we included a weighing function in our hologram calculation ${ }^{13}$. The input power of the incident laser beam is maintained in both cases so that any improvement is attributed to the beam shaping involved. 


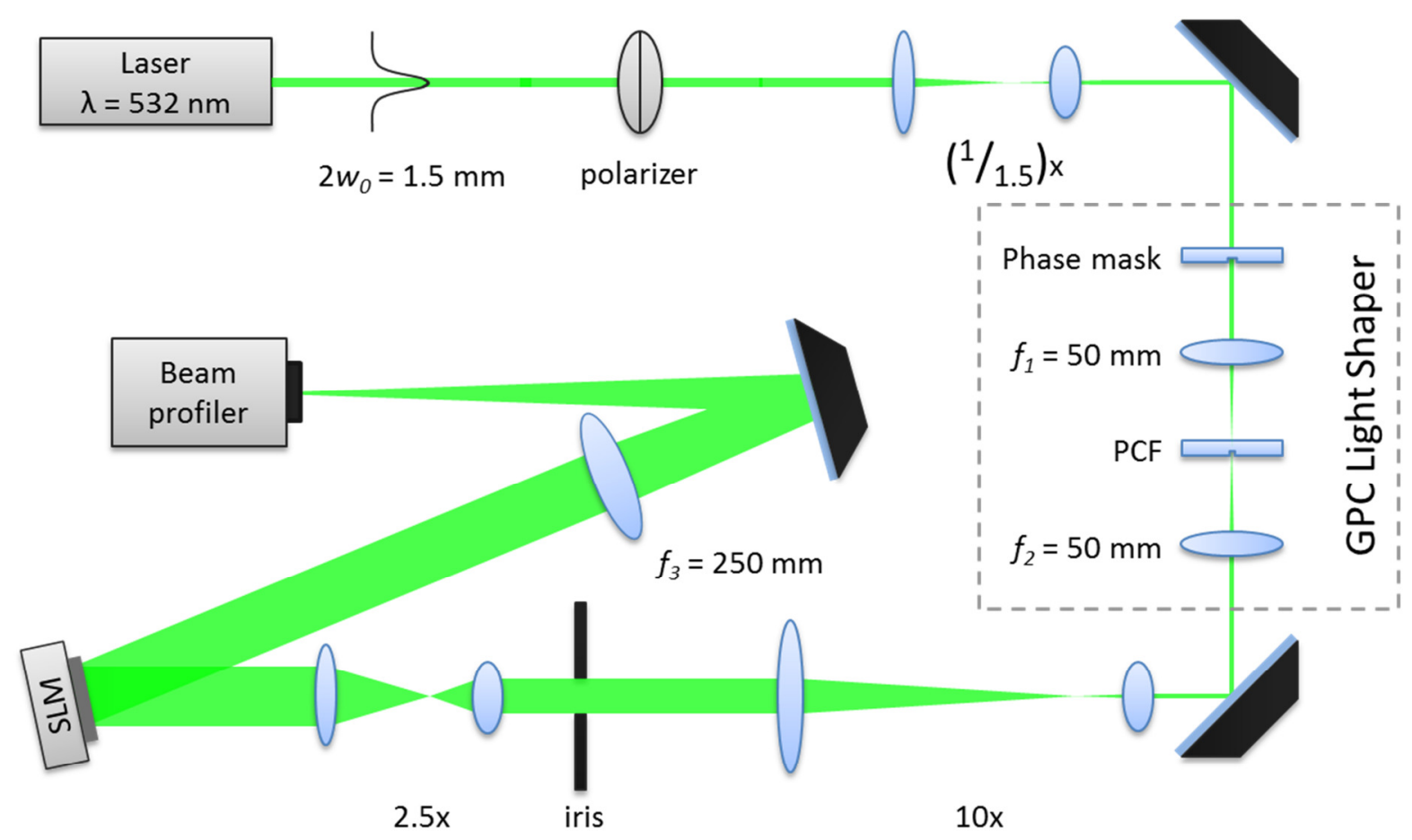

Figure 2. Phase-only diffractive setup. The GPC LS is placed before the SLM to perform preliminary beam shaping to match the shape of the modulation element of the SLM.

\section{RESULTS}

We use a random arrangement of 20,30 and 40 focal spots for our test patterns. We choose these patterns to simulate holographic optical tweezers scenarios and in multi-site photostimulation. Our results presented in figure 3 shows $\sim 3 \mathrm{x}$ gain when the GPC LS is used together with the holography setup compared with hard-truncated beam. The gain is calculated as the ratio of the average light spot intensity in the GPC-enhanced pattern to the average light spot intensity in the corresponding hard-truncated pattern.

Qualitatively, the GPC-enhanced focal spots are brighter than their hard-truncated counterparts. As an example, the spots in the 40 spot-pattern are still brighter compared to the spots in the hard-truncated case for the 20 spot-pattern. This suggests that we can generate more intense focal spots that can be used for multiple holographic optical tweezers and in multi-site photolysis. 

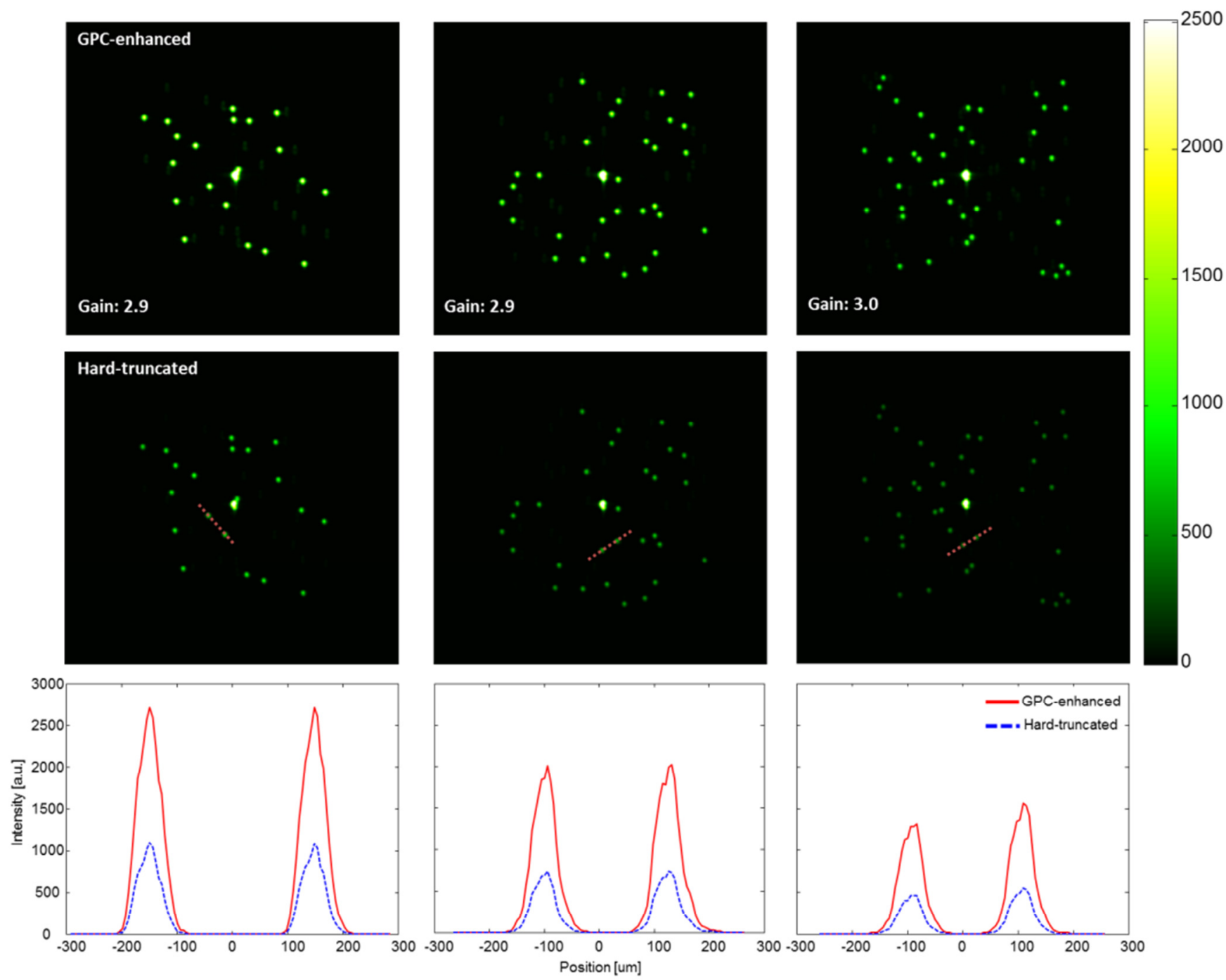

Figure 3. Experimental results showing the GPC-enhanced focal spots are $\sim 3 \mathrm{x}$ brighter than the hard-truncated counterpart.

The presence of speckles is an undesirable feature of holography and thus limits the application of extended patterns. However, for application where the aim is to efficiently generate high intensities such as in single-exposure two-photon polymerization ${ }^{14,15}$ this can be tolerated. We use binary images of the university's logo and a standard test image as our target pattern as shown in figure 4. A visual comparison of the experimental result for extended patterns using the GPC LS and hard-truncated beams are presented in figure 5. 

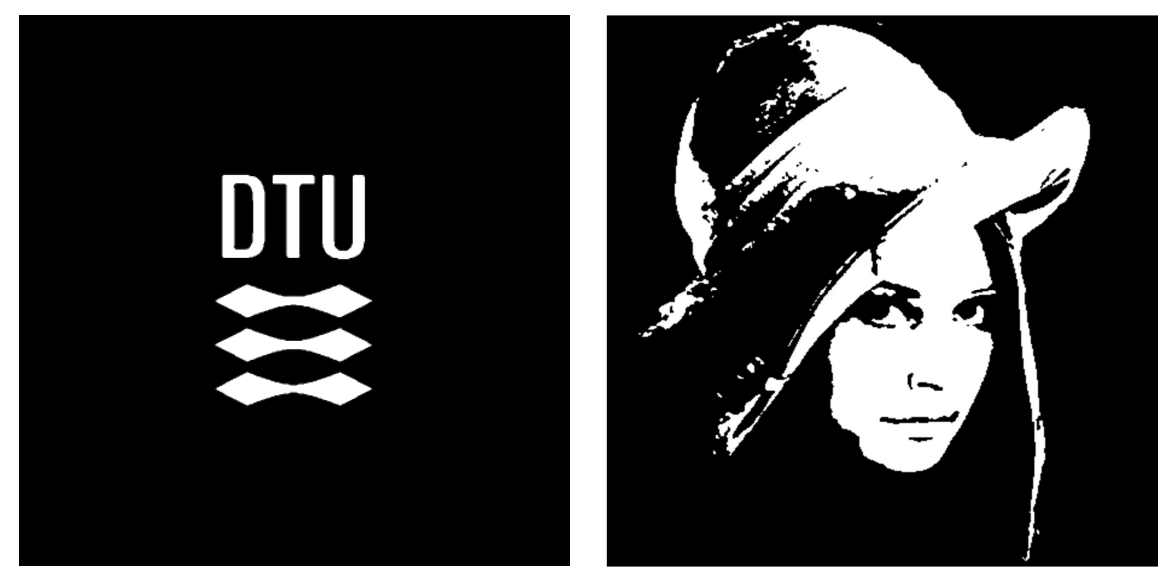

Figure 4. Binary images used as targets for the extended patterns. Left: Technical University of Denmark's logo. Right: Image of Lena.
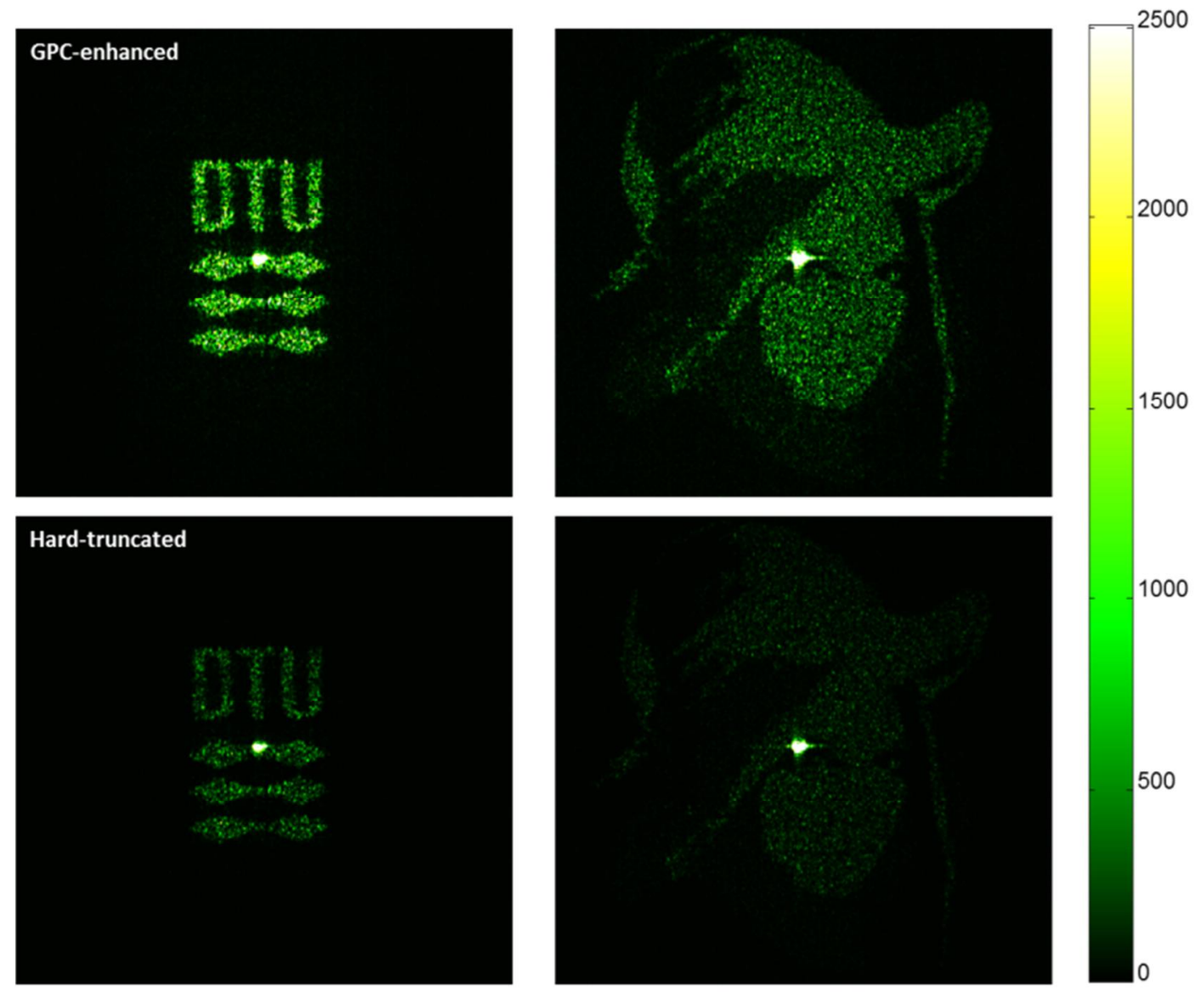

Figure 5. Experimental results for the extended patterns. GPC-enhanced extended patterns are also brighter compared to the hard-truncated counterpart.

\section{CONCLUSION}

We have shown an efficient illumination of a spatial light modulator using the GPC light shaper. Our results show that preliminary beam shaping using the GPC LS significantly increases the intensity of the resulting diffractive patterns by 
up to $\sim 3 \mathrm{x}$ compared to hard-truncated beams. This has been demonstrated for a number of focal spots and extended patterns. The GPC LS is not limited to devices such as SLMs since the phase mask can be fabricated to match arbitrary shapes and thus can also be used in microlens arrays that have inherent dead spaces between elements.

\section{ACKNOWLEDGMENTS}

This work is supported by Enhanced Spatial Light Control in Advanced Optical Fibres (e-space), a project financed by Innovation Fund Denmark and the Copenhagen Cleantech Cluster (CCC) for the GAP funding. We also acknowledge the support from Hamamatsu Photonics.

\section{REFERENCES}

[1] Liesener, J., Reicherter, M., Haist, T., Tiziani, H. J., "Multi-functional optical tweezers using computergenerated holograms," Opt. Commun. 185, 77-82 (2000).

[2] Curtis, J. E., Koss, B. A., Grier, D. G., "Dynamic holographic optical tweezers," Opt. Commun. 207, 169-175 (2002).

[3] Padgett, M., Bowman, R., "Tweezers with a twist," Nat. Photonics 5(6), 343-348 (2011).

[4] Chattrapiban, N., Rogers, E. A., Cofield, D., Hill, W. T., Roy, R., "Generation of nondiffracting Bessel beams by use of a spatial light modulator," Opt. Lett. 28(22), 2183-2185 (2003).

[5] Baumgartl, J., Mazilu, M., Dholakia, K., "Optically mediated particle clearing using Airy wavepackets,” Nat. Photonics 2, 675-678 (2008).

[6] Go, M. A., Stricker, C., Redman, S., Bachor, H.-A., Daria, V. R., "Simultaneous multi-site two-photon photostimulation in three dimensions," J. Biophotonics 5(10), 745-753 (2012).

[7] Kato, J., Takeyasu, N., Adachi, Y., Sun, H.-B., Kawata, S., "Multiple-spot parallel processing for laser micronanofabrication," Appl. Phys. Lett. 86(4), 044102 (2005).

[8] Bañas, A., Kopylov, O., Villangca, M., Palima, D., Glückstad, J., "GPC light shaper: static and dynamic experimental demonstrations," Opt. Express 22(20), 23759-23769 (2014).

[9] Palima, D., Alonzo, C. A., Rodrigo, P. J., Glückstad, J., "Generalized phase contrast matched to Gaussian illumination,” Opt. Express 15(19), 11971-11977 (2007).

[10] Bañas, A., Palima, D., Villangca, M., Aabo, T., Glückstad, J., "GPC light shaper for speckle-free one-and twophoton contiguous pattern excitation," Opt. Express 22(5), 5299-5310 (2014).

[11] Glückstad, J., Palima, D., [Generalized Phase Contrast: Applications in Optics and Photonics], Springer Series in Optical Sciences (2009).

[12] Gerchberg, R. W., Saxton, W. O., "A practical algorithm for the determination of phase from image and diffraction plane pictures," Optik (Stuttg). 35(2), 237-246 (1972).

[13] Persson, M., Engström, D., Goksör, M., "Real-time generation of fully optimized holograms for optical trapping applications," Proc. SPIE Vol. 8097, 8097, K. Dholakia and G. C. Spalding, Eds., 80971H (2011).

[14] Bautista, G., Romero, M. J., Tapang, G., Daria, V. R., "Parallel two-photon photopolymerization of microgear patterns," Opt. Commun. 282(18), 3746-3750, Elsevier B.V. (2009).

[15] Jeon, S., Malyarchuk, V., Rogers, J. A., Wiederrecht, G. P., "Fabricating three-dimensional nanostructures using two photon lithography in a single exposure step," Opt. Express 14(6), 2300-2308 (2006). 